\title{
New records of Ichneumonidae (Hymenoptera: Ichneumonoidea) from a coffee agroecosystem of southeastern Brazil
}

\author{
Daniell R. R. Fernandes ${ }^{1 \pm=}\left(\mathbb{0}\right.$, Rogéria I. R. Lara ${ }^{2}$, Nelson W. Perioto ${ }^{2}(\infty$ \\ ${ }^{1}$ Instituto Nacional de Pesquisas da Amazônia, Manaus, Amazonas, Brazil. ${ }^{2}$ Instituto Biológico, Ribeirão Preto, São Paulo, Brazil.

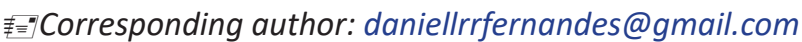

Edited by: Eduardo Amat

Received: August 07, 2020. Accepted: November 18, 2020. Published: December 01, 2020.

Abstract. We identified 614 specimens of Ichneumonidae (Hymenoptera: Ichneumonoidea) collected in a coffee agroecosystem located at Cravinhos, São Paulo, Brazil. As a result, 34 nominal species were found belonging to 22 genera and 10 subfamilies. Temelucha hilux Gauld, 2000 and Xiphosomella bonera Gauld, 2000 were registered for the first time in South America, Colpotrochia diabella Gauld \& Sithole, 2002 for the first time in Brazil, and Acerastes pertinax (Cresson, 1872), Colpotrochia mexicana (Cresson, 1868), Colpotrochia neblina Gauld \& Sithole, 2002, Colpotrochia texana (Cresson, 1872), Diplazon mulleolus Dasch, 1964, Eiphosoma nigrovittatum Cresson, 1865, Enicospilus flavus (Fabricius, 1775), Enicospilus glabratus (Say, 1835), Enicospilus purgatus (Say, 1835), Lymeon haemorrhoidalis (Taschenberg, 1876), Mesostenus alvarengae Porter, 1973, Microcharops plaumanni Gupta, 1987, Nonnus niger (Brullé, 1846), Ophiogastrella maculithorax Brues, 1912, Pachysomoides stupidus (Cresson, 1874), Polycyrtus albolineatus Cameron, 1911, and Trieces horisme Gauld \& Sithole, 2002 for the first time in the state of São Paulo. Other 14 species that are already registered for the state of São Paulo, were found for the first time in a coffee agroecosystem.

Keywords: Biological control, Coffea arabica, Darwin wasps, parasitoids.

Ichneumonidae is the largest family within Hymenoptera, currently divided into 39 subfamilies with more than 25,000 described species (Yu et al. 2016; Bennett et al. 2019). In general, they are solitary parasitoids, and most of the species attack larvae and pupae of Lepidoptera, Coleoptera, Neuroptera, Diptera and even Hymenoptera itself. This family is economically important as a biological control agent of pests from several cultures (Gauld 1991; Gauld 2006; Palacio \& Wahl 2006). In Brazil, the family is represented by 1,025 species distributed in 235 genera and 28 subfamilies (Fernandes et al. 2020a).

Despite its economic and ecological importance, this group of insects has not been adequately studied in most regions of Brazil; the majority of surveys have been conducted in Atlantic forest ecosystems (Kumagai \& Graf 2000; 2002; Guerra \& Penteado-Dias 2002; Kumagai 2002; Tanque et al. 2010; 2015), Cerrado (Pádua et al. 2014), Caatinga (Fernandes et al. 2019; Fernandes et al. 2020b), and specific agroecosystems, such as rubber tree (Hevea brasiliensis - Euphorbiaceae) (Tempest et al. 1998), green dwarf coconut trees (Cocos nucifera - Arecaceae) (Comério et al. 2012), organic farms (Sandonato et al. 2010) and guarana plantations (Paullinia cupana Sapindaceae) (Antunes \& Fernandes 2020).

In general, the assemblage of crop-associated parasitoids is determined by the features of the agroecosystem. Conventional cultures simplify the physical structure of the agricultural landscape, increasing the dependence on the use of agricultural inputs, and decreasing the diversity of parasitoid species (Estrada 2008). However, in less disturbed agroecosystems, without the intensive use of agricultural inputs, the parasitoid assembly is related to crop diversity, soil cover, weeds and vegetation adjacent to the crop (Estrada 2008; Sandonato et al. 2010).

The material examined was collected between May 2005 and April 2007 in a coffee plantation (Coffea arabica - Rubiaceae) at Cravinhos $\left(21^{\circ} 18^{\prime} 54^{\prime \prime} \mathrm{S} 47^{\circ} 47^{\prime} 39^{\prime \prime} \mathrm{W}\right)$, in the state of São Paulo, Brazil. The insects were sampled with: (a) Moericke traps - fixed on wooden stakes on the lower thirds of the plant (hereinafter referred as IYPT) and middle thirds (hereinafter referred as SYPT) of the plant and, (b) with two Jermy model light traps (hereinafter referred as LT) installed on poles about $50 \mathrm{~m}$ apart from each other (more details about sampling methodology see Lara et al. 2010 and Tango et al. 2014).

The material studied was deposited at LRRP - “Coleção Entomológica do Laboratório de Sistemática e Bioecologia de Parasitoides e Predadores do Instituto Biológico", Ribeirão Preto, São Paulo, Brazil (Curator: N. W. Perioto).

The geographic distribution of the species was verified with Mazón \& Bordera (2016) and Alvarado \& Figueroa (2019) for Metopiinae, Yu et al. (2016) for other ichneumonids, and with Fernandes et al. (2020a) for the occurrence in Brazilian states. New records for Brazil are indicated with an asterisk $(*)$, and for the state of São Paulo with two asterisks $(* *)$. The distribution maps were made using SimpleMappr online software (Shorthouse 2010).

In total, 1803 specimens of Ichneumonidae were collected, distributed in 16 subfamilies, 62 genera and 109 species/morphospecies. Of these, 614 specimens were analyzed, and 34 nominal species were identified belonging to 22 genera and 10 subfamilies. We have enlisted below only those specimens, which were identified to the species level. The other 1189 specimens were identified only in morphospecies until now and are not listed here.

Two species were found for the first time in South America, three in Brazil, and 20 in the state of São Paulo. Fourteen species already registered for the state of São Paulo are, for the first time, being documented in a coffee agroecosystem.

Brachycyrtinae

Brachycyrtus cosmetus (Walkley, 1956)

Material examined. SYPT, 01.xii.2005, [1 female].

Distribution. Brazil (MT, MG, PB, RR, SP), Costa Rica, Mexico, Suriname, Trinidad \& Tobago and Venezuela.

\section{Campopleginae}

Campoletis flavicincta (Ashmead, 1890)

Material examined. SYPT, 22.vii.2005, [1 male]; LT, 28.vii.2005, [1 male]; LT, 17.viii.2005, [1 male]; IYPT, 14.ix.2005, [1 female]; SYPT, 14.ix.2005, [1 female]; LT, 14.ix.2005, [1 male]; SYPT, 10.x.2005, [1 male]; IYPT, 09.xi.2005, [1 female]; IYPT, 22.xii.2005, [1 female]; SYPT, 
16.iii.2006, [1 female]; IYPT, 28.xii.2006, [2 males]; SYPT, 10.i.2007, [1 female]; SYPT, 07.ii.2007, [1 female].

Distribution. Canada, Honduras, India, Mexico, Nicaragua, Pakistan, Peru, United States of America, Uruguay and Brazil (MG, SP).

Microcharops peronatus (Cameron, 1911)

Material examined. IYPT, 14.vii.2005, [1 female]; SYPT, 05.x.2005, [1 male]; SYPT, 31.iii.2006, [2 females]; IYPT, 08.vi.2006, [1 male]; SYPT, 10.i.2007, [1 female]; SYPT, 28.ii.2007, [1 female].

Distribution. Argentina, Brazil (CE, GO, MG, RJ, SP, PR, SC), Colombia, Costa Rica, Ecuador, Guyana, Panama and Suriname.

Microcharops plaumanni Gupta, 1987

Material examined. IYPT, 24.xi.2005, [1 female].

Distribution. Brazil (MT, SC, SP**).

\section{Cremastinae}

Eiphosoma dentator (Fabricius, 1804)

Material examined. LT, 31.viii.2005, [1 male]; LT, 05.x.2005, [1 male]; LT, 19.x.2005, [1 male]; LT, 06.iv.2006, [1 male].

Distribution. Argentina, Barbados, Brazil (AM, MS, SP), Canada, Costa Rica, Cuba, El Salvador, Grenada, Guatemala, Guyana, Jamaica, Mauritius, Mexico, Paraguay, Peru, Puerto Rico, St. Vicente, Trindade \& Tobago, United States of America and Venezuela.

Eiphosoma laphygmae Costa Lima, 1953

Material examined. SYPT, 05.v.2005, [1 female]; LT, 12.v.2005, [1 female]; SYPT, 02.vi.2005, [1 male]; SYPT, 30.vi.2005, [1 female]; IYPT, 24.viii.2005, [2 males]; IYPT, 14.ix.2005, [1 female]; SYPT, 21.ix.2005, [1 female]; IYPT, 30.ix.2005, [2 females]; LT, 05.x.2005, [2 females]; SYPT, 10.x.2005, [1 male]; LT, 02.xi.2005, [1 female]; SYPT, 09.xi.2005, [1 male]; SYPT, 01.xii.2005, [1 male]; SYPT, 08.xii.2005, [1 male]; IYPT, 29.xii.2005, [1 female]; SYPT, 29.xii.2005, [2 females]; SYPT, 02.ii.2006, [2 males]; LT, 02.ii.2006, [2 females and 3 males]; IYPT, 9.ii.2006, [1 male]; LT, 9.ii.2006 [1 male]; LT, 15.ii.2006, [1 female]; IYPT, 9.iii.2006, [1 male]; SYPT, 31.iii.2006, [1 female]; SYPT, 6.iv.2006, [1 female]; SYPT, 27.iv.2006, [1 female and 1 male]; LT, 1 female, 27.iv. 2006, L; IYPT, 4.v.2006, [1 male]; IYPT, 2.vi.2006, [2 females and 1 male]; IYPT, 8.vi.2006, [1 female]; LT, 23.xi.2006, [1 male]; SYPT, 28.ii.2007, [1 male]; SYPT, 7.iii.2007, [1 female]; IYPT, 14.iii.2007, [1 male]; LT, 22.iii.2007, [1 female]; IYPT, 29.iii.2007, [1 female]; SYPT, 29.iii.2007, [1 female]; LT, 29.iii.2007, [1 female and 1 male]; SYPT, 4.iv.2007, [1 male].

Distribution. Belize, Bolívia, Brazil (MA, GO, MG, RJ, MS, SP), Colombia, Costa Rica, Cuba, Honduras, Mexico and Venezuela.

\section{Eiphosoma nigrovittatum Cresson, 1865}

Material examined. SYPT, 14.ix.2005, [1 male].

Distribution. Belize, Brazil (MS, ES, SP**), Costa Rica, Cuba, Guatemala, Jamaica, Mexico, Peru, Puerto Rico, Trinidad \& Tobago, United States of America and Venezuela.

Pristomerus spinator (Fabricius, 1804)

Material examined. LT, 05.x.2005, [1 female]; SYPT, 06.ix.2006, [3 females].

Distribution. Brazil (SP), Canada, Costa Rica, Guyana, Honduras, Mexico, Nicaragua and United States of America.

Temelucha hilux Gauld, 2000 (Fig. 1)

Material examined. SYPT, 9.xi.2006, 1 [female]; LT, 10.i.2007, [1 female].

Distribution. Brazil* (SP**), Costa Rica and Mexico.

Xiphosomella bonera Gauld, 2000 (Fig. 1)

Material examined. 1 female. SYPT, 22.vi.2005, [1 female].

Distribution. Brazil* (SP**) and Costa Rica.

\section{Cryptinae}

Acerastes pertinax (Cresson, 1872)
Material examined. IYPT, 23.xi.2006, [1 male].

Distribution. Brazil (RJ, SP**, SC), Cuba, Honduras, Jamaica, Mexico, Panama, Paraguay, Puerto Rico, Trinidad \& Tobago, United States of America and Venezuela .

Lymeon haemorrhoidalis (Taschenberg, 1876)

Material examined. YPT, 22.vii.2005, [1 male]; SYPT, 15.ii.2006, [1 female]; IYPT, 31.iii.2006, [1 female]; IYPT, 27.iv.2006, [1 female]; SYPT, 4.v.2006, [1 male].

Distribution. Brazil (MG, SP**, SC).

Mesostenus alvarengae Porter, 1973

Material examined. SYPT, 5.v.2005, [1 female and 1 male]; IYPT, 12.v.2005, [1 female]; SYPT, 12.v.2005, [2 females and 1 male]; IYPT, 2.vi.2005, [1 female]; IYPT, 9.vi.2005, [2 males]; IYPT, 22.vi.2005, [1 male]; IYPT, 28.vii.2005, [1 female and 1 male]; IYPT, 3.viii.2005, [1 male]; SYPT, 3.viii.2005, [3 females]; SYPT, 14.ix.2005, [2 females]; SYPT, 21.ix.2005, [1 female]; IYPT, 26.x.2005, [1 female]; SYPT, 26.x.2005, [1 male]; IYPT, 16.xi.2005, [2 females and 2 males]; IYPT, 1.xii.2005, [2 females]; SYPT, 1.xii.2005, [2 females and 1 male]; IYPT, 8.xii.2005, [1 male]; SYPT, 8.xii.2005, [1 male]; IYPT, 15.xii.2005, [1 female]; IYPT, 29.xii.2005, [1 female]; SYPT, 29.xii.2005, [1 female]; IYPT, 12.i.2006, [1 male]; IYPT, 19.i.2006, [3 females and 1 male]; IYPT, 26.i.2006, [1 female]; SYPT, 26.i.2006, [2 females]; IYPT, 2.ii.2006, [1 female]; SYPT, 2.ii.2006, [1 female and 1 male]; IYPT, 9.ii.2006, [3 females]; SYPT, 9.ii.2006, [1 female and 1 male]; LT, 9.ii.2006, [1 male]; SYPT, 15.ii.2006, [2 females]; IYPT, 23.ii.2006, [2 females and 2 males]; IYPT, 3.iii.2006, [1 female and 1 male]; IYPT, 9.iii.2006, [3 females]; SYPT, 9.iii.2006, [2 females]; IYPT, 17.iii.2006, [4 females]; IYPT, 24.iii.2006, [3 females and 3 males]; IYPT, 31.iii.2006, [4 females and 4 males]; IYPT, 6.iv.2006, [3 females and 4 males]; SYPT, 6.iv.2006, [2 females and 1 male]; IYPT, 12.iv.2006, [2 females and 3 males]; LT, 12.iv.2006, [1 male]; SYPT, 4.v.2006, [1 female]; LT, 13.xii.2006, [1 female]; LT, 7.iii.2007, [1 male].

Distribution. Argentina and Brazil (PA, RJ, SP**).

Messatoporus variegatus (Szépligeti, 1913)

Material examined. IYPT, 30.ix.2005, [1 female].

Distribution. Argentina, Bolivia, Brazil (RO, BA, ES, SP, PR, SC), Trinidad \& Tobago and Uruguay.

Pachysomoides stupidus (Cresson, 1874)

Material examined. SYPT, 22.xii.2005, [1 female].

Distribution. Brazil (RJ, $\left.\mathrm{SP}^{* *}\right)$, Costa Rica, Mexico, Panama, United States of America and Venezuela.

Polycyrtus albolineatus Cameron, 1911

Material examined. SYPT, 26.x.2005, [1 female]; SYPT, 15.ii.2006, [1 male]; SYPT, 3.iii.2006, [1 female and 1 male]; IYPT, 16.iii.2006, [1 male]; SYPT, 31.iii.2006, [1 male]; SYPT, 6.iv.2006, [1 male]; IYPT, 12.iv.2006, [1 male].

Distribution. Bolivia, Brazil (PE, SP**) and Guyana.

\section{Diplazontinae}

Diplazon laetatorius (Fabricius, 1781)

Material examined. IYPT, 5.x.2005, [1 female]; IYPT, 9.xi.2005, [1 female]; SYPT, 6.iv.2006, [1 female]; SYPT, 13.vii.2006, [1 female]; LT, 13.vii.2006, [1 female]; SYPT, 20.vii.2006, [1 female].

Distribution. Worldwide, Brazil (RJ, SP, PR).

Diplazon mulleolus Dasch, 1964

Material examined. SYPT, 14.ix.2005, [1 female]; LT, 10.x.2005, [1 male].

Distribution. Brazil (SP**, SC).

\section{Metopiinae}

Colpotrochia diabella Gauld \& Sithole, 2002 (Fig. 1)

Material examined. LT, 26.i.2006, [1 male]. 
Distribution. Brazil* (SP**), Costa Rica, Mexico and Venezuela.

Colpotrochia mexicana (Cresson, 1868)

Material examined. LT, 19.i.2007, [1 male]

Distribution. Brazil (MG, SP**), Costa Rica and Mexico.

Colpotrochia neblina Gauld \& Sithole, 2002

Material examined. IYPT, 6.xii.2006, [1 female].

Distribution. Brazil (MG, SP**) and Costa Rica.

Colpotrochia texana (Cresson, 1872)

Material examined. IYPT, 5.x.2005, [1 male].

Distribution. Brazil (SP**, SC), Costa Rica, Ecuador, Mexico and United States of America.

Trieces horisme Gauld \& Sithole, 2002

Material examined. IYPT, 9.vi.2005, [1 female]; IYPT, 19.x.2006, [1 male].

Distribution. Argentina, Belize, Brazil (SP**, SC), Costa Rica, Ecuador, Mexico, Panama, Peru and Venezuela.

\section{Nesomesochorinae}

Nonnus niger (Brullé, 1846)

Material examined. SYPT, 24.iii.2006, [1 female].

Distribution. Bolivia, Brazil (GO, RJ, SP**, RS), Guyana, Panama and Peru.

\section{Ophioninae}

Enicospilus flavoscutellatus (Brullé, 1846)

Material examined. LT, 5.v.2005, [1 male]; SYPT, 20.iv.2006, [1 female and 1 male]; SYPT, 4.v.2006, [1 female]; SYPT, 9.xi.2006, [1 female]; LT, 20.xii.2006, [2 males].

Distribution. Argentina, Bolivia, Brazil (BA, ES, MS, RJ, SP, SC, RS), Colombia, Costa Rica, Cuba, Dominica, Dominican Republic, Ecuardor, Grenada, Guadaloupe, Guatemala, Guyana, Jamaica, Mexico, Panama, Peru, Puerto Rico, St. Kitts-Nevis, St. Lucia, Suriname and Venezuela.

\section{Enicospilus flavostigma Hooker, 1912}

Material examined. LT, 31.iii.2006, [1 male].

Distribution. Argentina, Bahamas, Belize, Bolivia, Brazil (RN, BA, MT, MG, RJ, SP, SC), Canada, Chile, Colombia, Costa Rica, Cuba, Dominican Republic, Ecuador, El Salvador, French Guiana, Guatemala, Guyana, Mexico, Panama, Peru, Puerto Rico, Suriname, United States of America and Venezuela.

Enicospilus flavus (Fabricius, 1775)

Material examined. IYPT, 16.iii.2006, [1 male]; SYPT, 24.iii.2006, [1 male]; LT, 6.iv.2006, [1 male]; LT, 27.iv.2006, [1 male]; IYPT, 11.iv.2007, [1 male]; LT, 11.iv.2007, [1 male].

Distribution. Anguilla, Argentina, Bahamas, Bermuda, Bolivia, Brazil (RN, BA, ES, SP**, SC), Colombia, Cuba, Dominican Republic, Ecuador, Grenada, Guatemala, Guyana, Haiti, Jamaica, Mexico, Nicaragua, Panama, Paraguay, Peru, Puerto Rico, St. Kitts-Nevis, St. Lucia, St. Vicent, Suriname, United States of America, Venezuela and Virgin Islands.

\section{Enicospilus glabratus (Say, 1835)}

Material examined. LT, 20.iv.2006, [1 female and 2 males]; LT, 27.iv.2006, [1 male]; IYPT, 4.v.2006, [1 male]; LT, 25.v.2006, [1 male].

Distribution. Argentina, Bahamas, Belize, Bolivia, Brazil (BA, GO, MS, RJ, SP**, PR, SC, RS), Canada, Colombia, Costa Rica, Cuba, Dominican Republic, Ecuador, Guatemala, Jamaica, Mexico, Netherlands Antilles, Panama, Paraguay, Peru, Puerto Rico, Trinidad \& Tobago, United States of America, Venezuela and Virgin Islands.

Enicospilus purgatus (Say, 1835)

Material examined. LT, 16.vi.2005, [1 male ]; LT, 24.xi.2005, [1 female]; LT, 29.xii.2005, [1 male]; LT, 19.i.2006, [1 female]; LT, 3.iii.2006, [1 female]; LT, 31.iii.2006, [1 female]; LT, 6.iv.2006, [1 male]; LT, 12.iv.2006, [1 male]; LT, 27.iv.2006, [1 male]; LT, 6.vii.2006, [1 female]; LT, 20.xii.2006, [1 female]; LT, 28.xii.2006, [1 female]; LT, 23.ii.2007, [1 female].

Distribution. Antigua, Argentina, Bermuda, Bolivia, Brazil (AM, BA, MG, SP**, PR, SC), Canada, Colombia, Costa Rica, Cuba, Dominican Republic, Ecuador, Guyana, Jamaica, Juan Fernandez Islands, Mexico, Nicaragua, Panama, Peru, Puerto Rico, St. Vicent, Trinidad \& Tobago, United States of America, Venezuela and Virgin Islands.

Enicospilus trilineatus (Brullé, 1846)

Material examined. SYPT, 5.v.2005, [2 males]; IYPT, 16.III.2006, [1 female].

Distribution. Argentina, Bahamas, Belize, Bermuda, Bolivia, Brazil (PA, CE, PE, BA, MT, MG, ES, RJ, SP, PR, SC, RS), Chile, Colombia, Costa Rica, Cuba, Dominican Republic, Ecuador, El Salvador, Galapagos Islands, Grenada, Guatemala, Guyana, Haiti, Honduras, Jamaica, Martinique, Mexico, Montserrat, Panama, Paraguay, Peru, Puerto Rico, St. Lucia, St. Vicent, Suriname, Trinidad \& Tobago, United States of America and Venezuela.

Ophiogastrella maculithorax Brues, 1912

Material examined. LT, 21.ix.2005, [1 male]; LT, 25.x.2006, [1 female].

Distribution. Brazil (PB, SP**), Costa Rica and Mexico.

Ophion flavidus Brullé, 1846

Material examined. 101 females and 217 males were identified. This species was the most frequently collected in this study, with several specimens collected in IYPT, SYPT and LT, in all the months, between May 2005 and April 2007.

Distribution. Argentina, Brazil (AM, RN, MG, ES, MS, SP), Costa Rica, Cuba, Dominican Republic, Guatemala, Honduras, Mexico, Nicaragua, Panama, Paraguay, Peru, Puerto Rico, United States of America and Uruguay.

\section{Orthocentrinae}

\section{Chilocyrtus carinatus Townes, 1971}

Material examined. SYPT, 10.x.2005, [1 female]; IYPT, 16.xi.2005, [1 female]; IYPT, 26.i.2006, [1 female]; LT, 6.xii.2006, [1 male]; IYPT, 28.xii.2006, [1 female]; SYPT, 25.i.2007, [1 female]; SYPT, 14.ii.2007, [1 male].

Distribution. Argentina and Brazil (SP, PR, SC).

\section{Pimplinae}

Zaglyptus simonis (Marshall, 1892)

Material examined. IYPT, 5.v.2005, [1 male]; SYPT,12.v.2005, [1 male]; IYPT, 3.viii.2005, [1 male]; SYPT, 10.viii.2005, [2 females]; 17. IYPT, viii.2005, [1 male]; SYPT, 17.viii.2005, [1 male]; IYPT, 24.xi.2005, [1 female]; SYPT, 8.xii.2005, [1 male]; IYPT, 12.i.2006, [1 female]; IYPT, 19.i.2006, [1 male]; IYPT, 3.iii.2006, [1 female]; IYPT, 16.iii.2006, [1 male]; IYPT, 24.iii.2006, [1 male]; IYPT, 31.iii.2006, [1 male]; IYPT, 6.iv.2006, [1 female]; SYPT, 6.iv.2006, [1 male]; L, 6.iv.2006, [1 female]; IYPT, 12.iv.2006, [2 females]; SYPT, 12.iv.2006, [1 male]; IYPT, 20.iv.2006, [1 male]; IYPT, 27.iv.2006, [2 males]; IYPT, 25.v.2006, [1 female and 1 male]; IYPT, 16.vi.2006, [1 female]; SYPT, 25.x.2006, [1 female]; SYPT, 1.xi.2006, [1 female]; IYPT, 13.xii.2006, [1 male]; SYPT, 20.xii.2006, [1 male].

Distribution. Brazil (MG, SP, PR, SC), Costa Rica, Panama, Trinidad \& Tobago and Venezuela. 


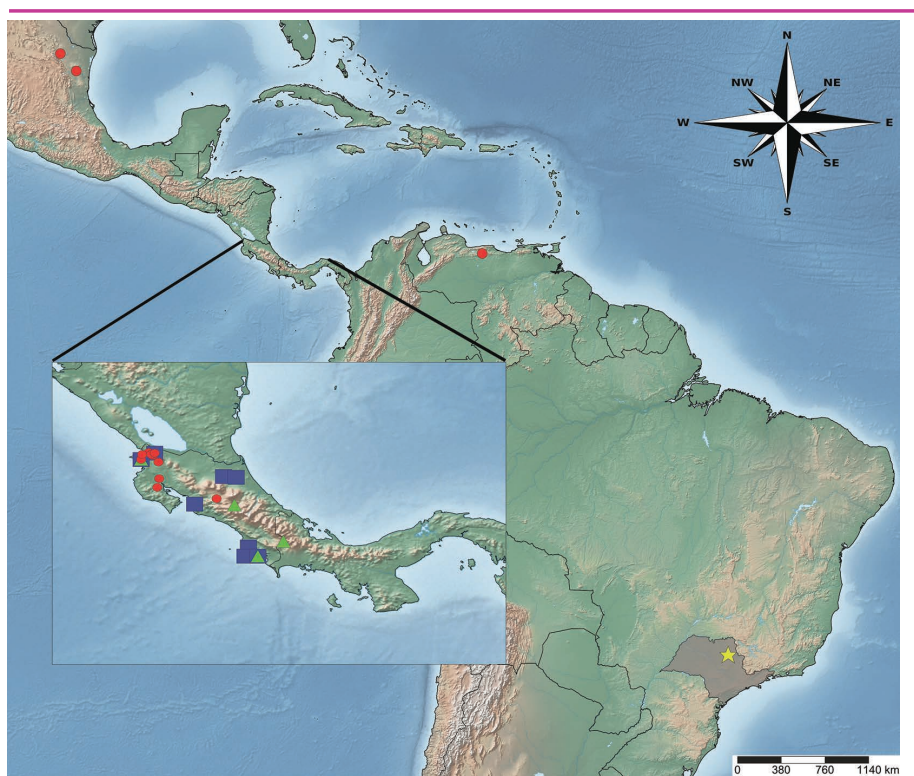

Figure 1. Distribution records of Colpotrochia diabella, Temelucha hilux and Xiphosomella bonera. Red circle = previous records of Colpotrochia diabella; green triangle = previous records of Temelucha hilux; blue square = previous records of Xiphosomella bonera; yellow star $=$ new records of Colpotrochia diabella, Temelucha hilux and Xiphosomella bonera; gray area = São Paulo state.

\section{Acknowledgments}

We would like to thank "Fundação de Amparo à Pesquisa do Estado do Amazonas" (FAPEAM), Edital no 030/2013 - Universal Amazonas (process no 062.00770/2015) and "Programa de Capacitação Institucional" - PCl/CNPq (process no 312879/2019-9) for funding. We also thank Ana Paula Silva Loffredo, Bernardo Ferreira dos Santos and Ivy Frizo de Melo for confirmation of Pimplinae, Cryptinae and Eiphosoma species respectivelly; Ahana Maitra for English revision and to anonymous reviewers for their valuable suggestions.

\section{Author's Contributions}

DRRF identified the specimens; DRRF, RIRL and NWP wrote the manuscript.

\section{References}

Alvarado, M.; Figueroa, L. (2019) New country records of the Metopiinae (Hymenoptera: Ichneumonidae) in the Neotropical region. Entomological Communications, 1, ec01010. doi: 10.37486/26751305.ec01010

Antunes, N. T. B.; Fernandes, D. R. R. (2020) Faunistic analysis of Ichneumonidae (Hymenoptera) in Guarana (Paullinia cupana) crop, with new records of genera for the Brazilian Amazon. Arquivos do Instituto Biológico, 87: e0832018. doi: 10.1590/1808-1657000832018

Bennett, A. M. R.; Cardinal, S.; Gauld, I. D.; Wahl, D. B. (2019) Phylogeny of the subfamilies of Ichneumonidae (Hymenoptera). Journal of Hymenoptera Research, 71: 1-156. doi: 10.3897/jhr.71.32375

Comério, E. F.; Onody, H. C.; Benassi, V. L. R. M. (2012) Levantamento da fauna de Ichneumonidae (Hymenoptera) em cultivo de coqueiro anão verde associado às plantas invasoras. EntomoBrasilis, 5(2): 109-114. doi: 10.12741/ebrasilis.v5i2.196

Estrada, C. I. N. (2008) Control biológico de insectos: um enfoque agroecológico. Medellín: Editorial Universidad de Antioquia.

Fernandes, D. R. R.; Antunes; N. T. B.; Araujo, E. L.; Lara, R. I. R.; Perioto, N. W. (2020b) Hymenoptera fauna, with emphasis on Ichneumonidae from an area of Caatinga in Northeast Brazil. EntomoBrasilis, 13: e0874. doi: 10.12741/ebrasilis.v13.e0874

Fernandes, D. R. R.; Santos, B. F.; Pádua, D. G.; Araujo, R. O. (2020a) Ichneumonidae. In: Catálogo Taxonômico da Fauna do Brasil. PNUD. Available in: <http://fauna.jbrj.gov.br/fauna/faunadobrasil/2248>. Access on: 05.ix. 2020
Fernandes, D. R. R.; Santos, J. J. M.; Lara, R. I. R.; Silva Junior, J. C.; Ferreira, H. A.; Perioto, N. W. (2019) Fauna de Ichneumonidae (Hymenoptera: Ichneumonoidea) em áreas de Caatinga do Sudoeste da Bahia, Brasil. EntomoBrasilis, 12(3): 126-131. doi: 10.12741/ebrasilis.v12i3.837

Gauld, I. D. (1991) The Ichneumonidae of Costa Rica, 1. Memoirs of the American Entomological Institute, 47: 1-589.

Gauld, I. D. (2006) Familia Ichneumonidae. In: Hanson, P. E.; I. D. Gauld (eds.), Hymenoptera de la Region Neotropical, pp. p. 446-487. Memoirs of the American Entomological Institute, 77: 1-994.

Guerra, T. M.; Penteado-Dias, A. M. (2002) Abundância de Ichneumonidae (Hymenoptera) em área de mata em São Carlos, Estado de São Paulo, Brasil. Acta Scientiarum: Biological Sciences, 24(2): 363-368.

Kumagai, A. F.; Graf, V. (2000) Ichneumonidae (Hymenoptera) de áreas urbana e rural de Curitiba, Paraná, Brasil. Acta Biológica Paranaense, 29(1-4): 153-168.

Kumagai, A. F.; Graf, V. (2002) Biodiversidade de Ichneumonidae (Hymenoptera) e monitoramento das espécies de Pimplinae e Poemeniinae do Capão da Imbuia, Curitiba, Paraná. Revista Brasileira de Zoologia, 19(2): 445-452. doi: 10.1590/S010181752002000200010

Kumagai, A. F. (2002) Os Ichneumonidae (Hymenoptera) da Estação Ecológica da Universidade Federal de Minas Gerais, Belo Horizonte, com ênfase nas espécies de Pimplinae. Revista Brasileira de Entomologia, 46(2): 189-194. doi: 10.1590/S008556262002000200011

Lara, R. I. R.; Perioto, N. W.; Freitas, S. (2010) Diversidade de hemerobiídeos (Neuroptera) e suas associações com presas em cafeeiros. Pesquisa Agropecuária Brasileira, 45(2): 115-123. doi: 10.1590/S0100-204X2010000200001

Mazón, M.; Bordera, S. (2016) Description of the first species of Trieces (Hymenoptera: Ichneumonidae) with tyloids in the male antennae and new records of Neotropical species. Neotropical Entomology, 45: 280-287. doi: 10.1007/s13744-016-0381-2

Pádua, D. G.; Zampieron, S. L. M.; Nunes, J. F. (2014) Composition of the families of parasitoids wasp in the Parque Nacional da Serra da Canastra, Minas Gerais, Brazil. EntomoBrasilis, 7(3): 199-206. doi: 10.12741/ebrasilis.v7i3.443

Palacio, E. E.; D. B. Wahl (2016) Familia Ichneumonidae. In: Fernández, F.; Sharkey, M.J. (eds.), Introducción a los Hymenoptera de la Región Neotropical, pp. 293-330. Bogotá: Sociedad Colombiana de Entomología y Universidad Nacional de Colombia.

Sandonato, D. L.; Onody, H. C.; Penteado-Dias, A. M. (2010) Fauna de Campopleginae (Hymenoptera, Ichneumonidae) em hortas orgânicas em Araraquara e São Carlos, SP, Brasil. Biota Neotropica, 10(2): 117-121. doi: 10.1590/S1676-06032010000200014

Shorthouse, D. P. (2010) SimpleMappr, an online tool to produce publication-quality point maps. http://www.simplemappr.net. Access on: 20.x.2020.

Tango, M. F. A.; Fernandes, D. R. R.; Paz, C. C. P.; Lara, R. I. R.; Perioto, N. W. (2014) Orgilinae (Hymenoptera: Braconidae) en cultivo de café de Cravinhos, SP, Brasil. Revista Colombiana de Entomología, 40(1): 25-33.

Tanque, R. L.; Kumagai, A. F.; Frieiro-Costa, F. A.; Souza, B. (2010) Ichneumonidae (Insecta: Hymenoptera) da Reserva do Boqueirão, Ingaí - MG. Revista Brasileira de Zoociência, 12(3): 241-247.

Tanque, R. L.; Kumagai, A. F.; Souza, B.; Korasaki, V. (2015) Structure and dynamics of the taxocenoses of Pimplinae, Poemeniinae, Rhyssinae, Anomaloninae and Metopiinae in an urban secondary semideciduous montane forest. Neotropical Entomology, 44(3): 224-231. doi: 10.1007/s13744-015-0275-80

Tempest, A. M.; Bergmann, E. C.; Faria, A. M; Penteado-Dias, A. M; Imenes, S. D. L.; Guerra, T. M. (1998) Sobre os Ichneumonidae presentes em cultura de seringueira (Hevea brasiliensis). Arquivos do Instituto Biológico, 65(2): 63-68.

Yu, D. S.; Van Achterberg, C.; Horstmann, K. (2016) World Ichneumonoidea 2015. Ottawa: Taxapad. Database on flash-drive. Access on: 18.x.2019. 